\section{TOTAL HIP REPLACEMENT}

\author{
Dr. G. A. ROSSET \\ M.B., B.Ch. (Witwatersrand), F.R.C.S. (Edin.),
}

Consulting Orthopaedic Surgeon, Medical School, University of Witwatersrand and General Hospital, Johannesburg.

Degenerative arthritis of the hip joint, from whatever cause, is treated conservatively during the initial phases of the disease. The principles of treatment are to diminish joint loading, improve stability and range of movement and medication to lessen pain and the coexistent synovitis.

Reconstruction of the hip joint is recommended when pain, stiffness, swelling and deformity lead to decreasing functional ability. Clincally, joint destruction is accompanied by loss of stability and limitation of movement.

Today the most satisfactory form of reconstruction is by total replacement of the hip joint. Numerous designs are available but. in the Orthopaedic Unit of the Johannesburg Hospital preference is for the Charnley type of low friction arthroplasty. This unit comprises a plastic (high density poly-ethylene) acetabular cup articulating with a stainless steel femoral component.

The operation, in the majority of cases, is reserved for elderly people. Consequently the risks are increased, necessitating particular care in pre-operative assessment and post-operative rehabilitation. Particular attention is paid to prevent complications such as deep vein thrombosis, pulmonary embolism, chest infection and heart failure.

Under general anaesthesia with the patient in the supine position a lateral approach is employed. The fascia lata is incised in the same plane, exposing the greater trochanter.

The greater trochanter is osteotomised and mobilised superiorly with the attached gluteus medius and short external rotator muscles. The advantages of this step are that: (a) it gives a particularly good view of the hip joint and (b) it allows improvement of stability subsequently as, at the conclusion of the operation the trochanter is replaced more distally on the femur giving a greater mechanical advantage to the abductor muscles. A possible disadvantage is that firm bony union of the trochanter takes approximately 12 weeks and detachment during the rehabilitation period is a possibility. In practise this is a rare occurrence.

The hip is dislocated by flexing, adducting and externally rotating the leg. The head of the femur is removed thus exposing the acetabulum in its entirety. The acetabulum is prepared by deepening and expanding its margins with special reamers. Any residual cartilage is removed and key holes are drilled into the bony pelvis. The cup is subsequently accurately cemented into position. The importance of accurate positioning cannot be over emphasised; the cup must lie within the confines of the prepared acetabulum at an angle of $45^{\circ}$ to the horizontal plane and must be neither ante or retroverted. Poor position of the cup may lead to dislocation, instability and even loosening.

The femoral shaft is then reamed in order to accept the femoral prosthesis. Prior to cementing the femoral component into the shaft a trial reduction is performed to assess the range of movement. . Osteophytes which may impede movement and act as fulcra which assist dislocation are removed. The prosthesis is then accurately cemented into position paying particular attention to its position; no ante or retroversion being permitted. The cementing substance used for both components is poly methyl methacrylate which is basically an acrylic cement. Residual cement is removed and the new hip joint is now located.

The greater trochanter is re-attached to the shaft of the femur, being securely fixed with horizontal and vertical wires. The wound is closed in layers with suction drainage. These are important features of the operation not only to attain primary wound healing but particularly to prevent wound dehissance and haematoma formation which are particularly prone to turn septic. Infection would imply total failure of the operation.

The patient is carefully transferred to his bed maintaining the operated limb in abduction avoiding possible dislocation.

Blood loss during the operation amounts to approximately $1000 \mathrm{cc}$ and this is replaced intra operatively by intra venous infusion. The operative time is kept to a minimum, 60-80 minutes, in the knowledge that infection rates are increased in prolonged procedures. Post operatively prophylactic intravenous antibiotics are employed for $\mathbf{4 8}$ hours and sub anti-coagulant doses of Heparin are administered for 5 days.

The importance of a good exposure is stressed, associated with precise surgical technique, minimum operating time and above all attention to pre- and post-operative detail. Although the results of total hip replacement are impressive there are still sufficient complications to suggest that the procedure should be reserved for suitable patients. The operation should be done by qualified surgeons and the care and rehabilitation of such patients should be in the hands of nurses and physiotherapists who have been adequately exposed to the treatment of such cases. 